PHARMACOLOGY; ADVERSE EFFECTS

\section{Doxycycline induced intracranial hypertension}

Lochhead J, Elston JS BMJ 2003; 326: 641-642

This is a report of 2 patients taking doxycycline for malaria prophylaxis, one of whom suffered permanent damage to the eyes.

Doxycycline is widely used as an adjunctive periodontal treatment, and has been marketed for long-term use in low dosage (20 $\mathrm{mg} /$ dy). Intracranial hypertension (IH) has been reported as an adverse effect for other tetracyclines, and sometimes referred to as 'benign'. The first case involved a $21 \mathrm{yr}$-old female who had taken $100 \mathrm{mg}$ doxycycline daily for 3 weeks. She complained of headaches and blurred vision. IH was diagnosed and treated with acetazolamide, but was still present after 3 weeks, and treatment was continued a further 2 months at full dose and then reduced. This produced relief of symptoms and normal optic discs.

A second patient on doxycycline for 4 months developed severe optic damage at the end of this time, preceded by mild headaches and vomiting. She suffered optic atrophy, poor colour vision and visual acuity and an estimated $70 \%$ loss of vision. A leader in the same issue of the journal draws attention to these case histories, and warns that the disorder should be considered whenever anyone taking doxycycline begins to complain of a new headache. Such patients should stop the drug immediately, and be referred for diagnosis and treatment of intracranial hypertension and visual disorders if present.

\section{PREVENTIVE DENTISTRY; SOCIOLOGY}

\section{The power of children over adults when obtaining sweet snacks}

Roberts BP, Blinkhorn AS et al. Int J Paediatr Dent 2003; 13: 76-84

Access to money allows children to thwart parental control.

A random sample of 340 children aged 7-8 yrs selected from the Manchester area was interviewed about sources of money, cariogenic food intake, and ability to influence decisions of adults in charge of them on this matter. Questionnaires about the same matters were sent to all parents, and 181 were returned.

Data from both children and adults suggested that child influence declined with parental age, but the adult data suggested this was due to their limiting cariogenic food access, while child data suggested younger children had easier access to money. Children of younger parents more often had an additional source of money, usually grandparents, often without parents' apparent knowledge.

\section{ORAL AND MAXILLOFACIAL SURGERY; EPIDEMIOLOGY}

\section{Heterogeneity of temporomandibular disorders: cluster and case-control analyses Velly AM, Philippe P et al. J Oral Rehabil2002; 29: 969-979}

This study suggests the existence of 3 subgroups with TMJ pain related to life disturbances and one pain-free but disabled group.

In 2 Montreal dental clinics, 162 patients diagnosed with TMJ dysfunction were categorized in respect of the nature of their pains and jaw function. Cluster analysis identified 4 subgroups, which were compared with 100 controls who included 17 patients with symptomless manifestation of clicking or movement limitation.

Two clusters, of 9\% and 43\% of patients respectively, when compared with controls, had greater tendencies to clench and grind teeth and experience depression. These groups were considered dysfunctional by the authors. Past orthodontic treatment and female gender were more frequent in another cluster of 34\%, and depression in a further 15\%. The last group was pain-free, but almost all experienced disc displacement.

\section{CARDIOLOGY; ENDODONTICS}

\section{Effect of electronic apex locators on cardiac pacemaker function}

Garofalo RR, Ede EN etal. J Endodon 2002; 28: 831-833

Only one electronic apex locator (EAL) caused slight interference.

Various dental devices interfere with pacemakers, and the number of patients with them is increasing. Although the EAL was introduced in 1962, no study has yet examined its effect on pacemakers. Manufacturers warn against EAL use in patients wearing pacemakers. EALs measure changed electrical resistance as the canal apex is approached, and their use can reduce X-ray exposure.

A pacemaker set at 60 pulses/min was directly connected (an extreme test) to 5 EALs in turn, with a $150 \Omega$ resistor to simulate a canal apex. The pacemaker alone served as negative control, and was linked to an ECG simulator as a positive control. The pacemaker was monitored by telemetry. Only one EAL and the positive control interfered with normal pacing. The authors did not consider the effect of the EAL in question to be significant, and advocate clinical trials to clarify these results. 